\title{
Lorenz Curves, Inequality, and Social Welfare Under Changing Population Composition
}

\section{David Lam, University of Michigan}

This paper analyzes the effects of population growth and other demographic changes on the distribution of income by examining Lorenz curves and generalized Lorenz curves produced from combinations of income distributions. The paper shows that when subpopulations have equal means, a number of simple conditions govern the position of the Lorenz curve for the combined population relative to the Lorenz curves for the subpopulations. These conditions become less regular when the subpopulations have uifferent means, and suggest that a combined distribution will almost never Lorenz-dominate an original distribution. Implications of the results for intertemporal comparisons of social welfare and inequality are discussed. The results are illustrated by comparing income distributions across generations in Brazil.

\section{INTRODUCTION}

The theoretical foundation for intertemporal and cross-national comparisons of income distributions has been strengthened by research exploiting the analogy between rankings of income distributions and rankings of probability distributions in the economics of uncertainty.' This literature has provided new justification for the use of Lorenz curves as the basis for inequality comparisons by establishing the relationship between Lorenz dominance and stochastic dominance. Specifically, this literature proves that for distributions with equal means, the condition that distribution A Lorenz dominates distribution $B$ is equivalent to the condition that distribution $B$ can be produced from distribution $A$ by a series of regressive transfers (analogous to mean preserving spreads), and to the condition that distribution $A$ has higher expected social welfare for any symmetric " $S$-concave" social welfare function. Shorrocks (1983) provides a valuable extension of the results to distributions with different means, proving that domi-

\footnotetext{
'The seminal contribution is Atkinson (1970), with extensions by Rothschild and Stiglitz (1973) and Dasgupta, Sen, and Starrett (1973). A v'seful survey of the results is provided by Kakwani (1980).
}

Address correspondence to Professor David Lam, Department of Economics. University of Michigan, Ann Arbor, MI 48109. 
nance in mean inflated "generalized Lorenz curves" is formally equivalent to second-order stochastic dominance, with all that this implies for rankings of expected social welfare. An important outcome of these results is thiat they have served as a foundation for analyzing the consistency between standard measures of inequality and particular classes of social welfare functions, as in Newbery (1970), Sheshinski (1972), and Fields and Fei (1976).

As this literature has proven, there is a straightforward correspondence between changes in Lorenz curves and changes in social welfare when there are redistributions of income in the population. Actual changes in income distributions, however, often occur for reasons other than the direct income transfers which provide the theoretical basis for welfare-based inequality comparisons. One important source of change in the distribution of income is changes in population composition due to migration or natural increase. A large class of demographic and economic phenomena can be represented as additions of new entrants into some initial population. This paper explores the implications of changing population somposition on inequality and social welfare by analyzing the general properties of Lorenz curves and generalized Lorenz curves produced from combinations of populations. The results have important implications for analysis of the economic effects of population growth, the distributional effects of which have been debated in both theoretical and empirical research. ${ }^{2}$

The paper begins by identifying the position of the generalized Lorenz curve for a combined population relative to the generalized Lorenz curves of two original subpopulations. It is shown that the generalized Lorenz curve for a combined distribution is always less than or equal to a weighted average of the generalized Lorenz curves for the original populations, always lies between the original curves if they do not intersect, and intersects each of the original curves exactly as many times as the original curves intersect. Drawing on Shorrocks' proof of the direct relationship between generalized Lorenz curves and second-order stochastic dominance, these results are used to analyze the apparent changes in social wel ire implied by changes in population composition over time.

The paper then analyzes the properties of standard Lorenz curves for combined distributions, demonstrating that the Lorenz curve for the combined population must be less than or equal to a weighted

${ }^{2}$ See Lam (1987) for a survey of this literature. 
average of the original Lorenz curves. Beyond this fundamental inequality the results for generalized Lorenz curves do not extend directly to standard Lorenz curves when the subpopulations have different means. Most importantly, the paper proves that the combined distribution will Lorenz-dominate the original distribution if and only if the entering population has the same mean as the original distribution and itself Lorenz-dominates the original distribution.

The theoretical results are illustrated by comparing the income distribution for Brazilian males with the hypothetical income distribution implied by assigning brys ages $0-15$ the incomes of their fathers. A literal comparison of the generalized Lorenz curves and standard Lorenz curves for the two populations implies an unambiguous deterioration in expected social welfare across generations, but an amtiguous change in income inequality. The paper concludes by pointing out the ambiguities inherent in welfare interpretations of intertemporal changes in the distribution of income under changing population composition.

\section{LORENZ CURVES, GENERALIZED LORENZ CURVES, AND SOCIAL WELFARE}

The Lorenz curve plots cumulative shares of income as a function of cumulative population shares when individuals are ranked in increasing order of income. Given an income distribution with probability density function $f(y)$, the horizontal axis for the Lorenz curve is the cumulative distribution function $F(\hat{y})=\int_{0}^{\hat{y}} f(y) d y$. The vertical axis is the first moment distribution function $\Phi(\hat{y})=\frac{1}{\mu} \int_{0}^{\hat{y}} y f(y) d y$, where $\mu$ is the mean of the distribution. Since $\frac{d F(y)}{d y}=f(y)$ and $\frac{d \Phi(y)}{d y}=$ $\frac{y f(y)}{\mu}$, a convenient property is that

$$
\Phi^{\prime}[F(y)] \equiv \frac{d \Phi[F(y)]}{d F(y)}=\frac{y}{\mu} .
$$

For some purposes, including analysis of the extreme points, it is analytically convenient and conceptually appropriate to recognize the discreteness of individuals in the distribution. The discrete analog to (1) is 


$$
\frac{\Phi\left[F\left(y_{k}\right)\right]-\Phi\left[F\left(y_{k-1}\right)\right]}{F\left(y_{k}\right)-F\left(y_{k-1}\right)}=\frac{y_{k}}{\mu},
$$

where $y_{k}$ is the income of the $k$ th person in the rank-ordered distribution. Since the marginal income is by construction nondecreasing in $F(y)$, the slope of the Lorenz curve is everywhere nondecreasing. Particular attention will be given to the slope of the Lorenz curve as $F \rightarrow 0$ and $F \rightarrow 1$. Following (2), the slope of the curve out of the origin is $\frac{y^{m i n}}{\mu}$, where $y^{m i n}$ is the income of the poorest person in the population, assumed to be nonnegative. The slope approaching the upper right hand corner is $\frac{y^{\max }}{\mu}$, where $y^{\max }$ is the income of the richest person in the population.

In addition to Lorenz curves as traditionally defined, this paper analyzes the "generalized Lorenz curve," defined by Shorrocks (1983) as $\mu \Phi(F)$, the conventional Lorenz curve scaled up by mean income. Note from (1) and the definition that the slope of Shorrocks' generalized Lorenz curve at some $\hat{F}$ is $y(\hat{F})$. Gi ven Shorrocks' demonstration of the relationship between generalized Lorenz curves and comparisons of social welfare for distributions with different means, the generalized Lorenz curve is interesting in its own right, and, as will be seen below, is a useful tool in analyzing combined distributions.

Since the cases being considered in this paper necessarily involve changing population size, social welfare comparisons will be based on per capita social welfare. The analysis thus assumes the existence of per capita social welfurc funictions $W$, defined over the probability density function of the income distribution such that $W\left(f_{i}\right)=P W\left(f_{i}\right)$ for any population size $P$. This is consistent with Shorrocks (1983), and assumes a restriction to social welfare functions which satisfy the "symmetry axiom for population"' defined by Dasgupta et al. (1973). A preference for equity is assumed, formalized by restricting attention to the class of symmetric $S$-concave social welfare functions, also defined in Dasgupta et al. (1973). Social welfare comparisons will draw on Shorrocks' (1983) Theorem 2, which establishes that

$$
W\left(f_{i}\right) \leq W\left(f_{j}\right) \forall W \text { iff } \mu_{i} \Phi_{i}(F) \leq \mu_{j} \Phi_{j}(F) \forall F,
$$

where $W$ is any $S$-concave social welfare function.

These assumptions about social welfare comparisons do not imply that a measure of per capita social welfare is the appropriate way to 
evaluate demographic changes such as increases or decreases in fertility. One of the fundamental issues underlying the analysis here, but hardly resolved by it, is how to make welfare evaluations of the introduction of new (possibly unborn) members into a population. This is a philosophical issue far beyond the scope of this paper, ${ }^{3}$ but the issue should be kept in mind in interpreting the results below.

The proofs of the equivalence between the rankings of income distributions by Lorenz curves or generalized Lorenz curves and the rankings by social welfare functions strengthen the theoretical motivation for intertemporal and cross-national comparisons of income distributions. Increased confidence in the economic content of such comparisons may be misplaced, however, if the purely demographic component of changes in income distributions is substantial. Changes in the income distribution due to differential fertility across income classes, for example, may be observationally equivalent to changes caused by progressive or regressive transfers. ${ }^{4}$ The welfare implications of differential fertility, however, are much less straightforward than the welfare implications of regressive transfers, suggesting that the clearer welfare interpretation of income distribution comparisons provided by Atkinson (1970) and Shorrocks (1983) may be partially illusory. The following sections clarify the problem by formally analyzing the changes in social welfare and inequality implied by the introduction of new individuals into an existing population.

\section{GENERALIZED LORENZ CURVES FOR COMBINED DISTRIBUTIONS}

Consider an initial population with an income distribution defined by the density function $f_{1}(y)$ to which we introduce a second population with income distribution $f_{2}(y)$, producing the combined density function $f_{3}(y)=p_{1} f_{1}(y)+\left(1-p_{1}\right) f_{2}(y)$, where $p_{1}$ is the first population's share in the combined populaticn. We seck to identify restrictions on the relative position of the combined population's Lorenz clirve and generalized Lorenz curve. This will make it possible to answer such questions as whether the combined population can ever have unambiguously higher or unambiguously lower expected social welfare than the original population and whether the combine, d population can have

\footnotetext{
${ }^{3}$ See Nerlove, Razin, and Sadka (1987) for a recent survey of the issue and important theoretical contributions.

${ }^{4}$ See Lam (1986).
} 
an unambiguously more equal or unambiguously less equal distribution of income than the original population.

Since the marginal incomes for each distribution at a given value on the c.d.f. are important to the results below, it follows by construction of the c.d.f. for the combined popui tion that

$$
\min \left[y_{1}(F), y_{2}(F)\right] \leq y_{3}(F) \leq \max \left[y_{1}(F), y_{2}(F)\right] .
$$

Recall from (1) that these marginal incomes are the slopes of the generalized Lorenz curves at a given $F$. The p.d.f. and c.d.f. for the combined distribution are simply weighted averages of the p.d.f.'s and c.d.f.'s of the original distributions, with weights equal to the population shares. It does not follow, however, that the (generalized) Lorenz curve for the combined population is a weighted average of the original (generalized) Lorenz curves. In fact, the position of the generalized Lorenz curve for the combined population is governed by the following fundamental inequality:

Theorem 1. $\mu_{3} \Phi_{3}(F) \leq p_{1} \mu_{1} \Phi_{1}(F)+\left(1-p_{1}\right) \mu_{2} \Phi_{2}(F) \forall F$, with the equality holding wherever $y_{3}(F)=y_{2}(F)=y_{1}(F)$.

Proof: Following Atkinson (1970), integration by parts implies that

$$
\mu_{i} \Phi_{i}(\hat{F})=y_{i} \hat{F}-\int_{0}^{y_{i}(\hat{F})} F_{i}(y) d y .
$$

Using this result and the fact tîat $F_{3}(y)=p_{1} F_{1}(y)+\left(1-p_{1}\right) F_{2}(y)$, the generalized Lorenz curve for the combined distribution can be reduced to

$$
\begin{aligned}
\mu_{3} \Phi_{3}(\hat{F}) & =p_{1} \mu_{1} \Phi_{1}(\hat{F})+\left(1-p_{1}\right) \mu_{2} \Phi_{2}(\hat{F}) \\
& +p_{1}\left[\hat{F}\left(y_{3}-y_{1}\right)-\int_{y_{1}}^{y_{3}} F_{1}(y) d y\right] \\
& +\left(1-p_{1}\right)\left[\hat{F}\left(y_{3}-y_{2}\right)-\int_{y_{2}}^{y_{3}} F_{2}(y) d y\right] .
\end{aligned}
$$

Regardless of the signs of $y_{3}-y_{1}$ and $y_{3}-y_{2}$, the two terms in brackets on the right-hand side of (6) must be nonpositive, establishing the inequality in Theorem $1 .^{5}$ Since the last two terms in (6) go to

\footnotetext{
${ }^{5}$ A similar result can be found using standard Lorenz curves in ar: a.spublished paper by Satchell (1984), who provides a considerably different proof.
} 
zero when $y_{3}=y_{2}=y_{1}$, a sufficient (but not necessary) condition to satisfy the equality in Theorem 1 is that $y_{3}=y_{2}=y_{1}{ }^{6}$.

If the two original generalized Lorenz curves do not intersect, Theorem 1 insures that the generalized Lorenz curve for the combined population never lies above the dominant generalized Lorenz curve. Given the result in (3), Theorem 1 has the following implications for social welfare comparisons:

$$
\text { If } W\left(f_{1}\right) \leq W\left(f_{2}\right) \forall W \text {, then } W\left(f_{3}\right) \leq W\left(f_{2}\right) \forall W \text {. }
$$

If distribut ion 2 has higher expected social welfare than distribution 1 for any $S$-concave social welfare function, a combination of the two distributions will never have higher expected welfare than distribution $2 .^{7}$ It will be proven below that "generalized Lorenz dominance" by one of the original distributions is a necessary and sufficient condition for the combined distribution to have unambiguously lower expected social welfare than the dominating original distribution. It cannot be proven based on Theorem 1, however. Indeed, the rankings implied by Theorem 1 are quite limited. In and of itself it implies little more than that the generalized Lorenz curve for the combined population can never lie above both of the original generalized Lorenz curves, with corresponding implications for welfare comparisons.

Further rankings can be established by identifying the relative positions of the three generalized Lorenz curves when the two original curves do not intersect.

Theorem 2. $\mu_{3} \Phi_{3}(\hat{F})\left\{\begin{array}{l}\leq \\ \geq\end{array}\right\} \mu_{i} \Phi_{i}(\hat{F}) \forall \hat{F} \quad$ iff $\quad \mu_{j} \Phi_{j}(\hat{F})\left\{\begin{array}{l}\leq \\ \geq\end{array}\right\} \mu_{i} \Phi_{i}(\hat{F})$ $\forall \hat{F}, i, j=1,2$.

Proof: Atkinson (1970) proves that for distributions with the same mean, Lorenz dominance is equivalent to second order stochastic dominance. Extending the result to the case of different means-and thus to generalized Loren` curves-the generalization of Atkinson's theorem is

\footnotetext{
${ }^{6}$ From (4), $y_{3}=y_{1}=y_{2}$ if $y_{1}=y_{2}$; so, recalling (1), the three marginal incomes will be equal if and only if the slopes of the generalized Lorenz curves are equal at $\hat{F}$.

"It is important to emphasize that there is no "diversification" involved in the combination of distributions concidered he: $\mathrm{e}$. The combinations are like pouring two lottery urns into a single urn, rather than offering linez $r$ combinations of draws from separate urns.
} 


$$
\begin{aligned}
& \mu_{i} \Phi_{i}(\hat{F})-\mu_{j} \Phi_{j}(\hat{F}) \\
& \geq 0 \forall \hat{F} \text { iff } \int_{0}^{\dot{y}}\left[F_{j}(y)-F_{i}(y)\right] d y \geq 0 \forall \tilde{y} .
\end{aligned}
$$

Evaluating (8) for the case of $\mu_{3} \Phi_{3}-\mu_{i} \Phi_{i}$, and using the fact that $F_{3}=p_{i} F_{i}+\left(1-p_{i}\right) F_{j}$, the condition reduces to

$$
\begin{aligned}
& \mu_{3} \Phi_{3}(\hat{F})- \mu_{i} \Phi_{i}(\hat{F}) \\
& \geq 0 \forall \hat{F} \quad \text { iff } \quad \int_{0}^{\tilde{y}}\left[F_{i}(y)-F_{j}(y)\right] d y \geq 0 \forall \tilde{y} .
\end{aligned}
$$

By (8), the right-hand side of (9) will be satisfied if and only if $\mu_{j} \Phi_{j}(\hat{F})$ $\geq \mu_{i} \Phi_{i}(\hat{F}) \forall \hat{F}$. Repeating the step with the inequality reversed leads directly to Theorem $2 .^{8}$

Theorem 2 imposes a number of restrictions on the relative position of the generalized Lorenz curve for the combined population. It implies, for example, that the generalized Lorenz curve for the combined population lies everywhere below one of the original generalized Lorenz curves if and only if it lies everywhere above the other generalized Lorenz curve. It follows that

$$
\begin{aligned}
& \mu_{1} \Phi_{1}(F) \leq \mu_{3} \Phi_{3}(F) \leq \mu_{2} \Phi_{2}(F) \forall F \quad \text { iff } \quad \mu_{1} \Phi_{1}(F) \\
& \leq \mu_{2} \Phi_{2}(F) \forall F .
\end{aligned}
$$

The generalized Lorenz curve for the combined distribution lies everywhere between the the original generalized Lorenz curves if and only if the original generalized Lorenz curves do not intersect. Given Shorrocks' result in (3), this in tum implies that

$$
W\left(f_{1}\right) \leq W\left(f_{3}\right) \leq W\left(f_{2}\right) \forall W \quad \text { iff } \quad W\left(f_{1}\right) \leq W\left(f_{2}\right) \forall W,
$$

where $W$ is any $S$-concave social welfare function.

Theorem 2 implies that, regardless of the relative positions of the original generalized Lorenz curves, it can never be the case that the generalized Lorenz curve for the combined population lies everywhere below both of the original generalized Lorenz curves. That is, it must always be true that

$$
\mu_{3} \Phi_{3}(\hat{F}) \geq \min \left[\mu_{1} \Phi_{1}(\hat{F}), \mu_{2} \Phi_{2}(\hat{F})\right] \text { for some } \hat{F} .
$$

While this result may not seem surprising, the same condition is not true for standard Lorenz curves, as will be shown below. Note that

\footnotetext{
${ }^{8}$ Note that (8) also leads directly to the result in (3), providing an alternative proof of Shorrocks' Theorem 2.
} 
(12) in turn implies that the combined distribution can never be unambiguously inferior to both of the original distributions in the sense of having lower expected social welfare for all $S$-concave social welfare functions.

\section{INTERSECTING GENERALIZED LORENZ CURVES}

A further corollary of Theorem 2 is that if the original generalized Lorenz curves intersect, then the generalized Lorenz curve for the combined distribution must intersect both of the original curves. This can be proven by noting that any counterexample is directly refuted by Theorem 2 . We can go further, however, in identifying "he relative positions of the generalized Lorenz curves in the case of intersections:

Theorem 3. If $\mu_{1} \Phi_{1}$ and $\mu_{2} \Phi_{2}$ intersect $n$ times, then $\mu_{3} \Phi_{3}$ intersects each of the original curves exactly $n$ times.

Proof: The first step is to prove that $\mu_{3} \Phi_{3}$ must cross each original curve at least once for every crossing of the original curves. Denote the value of $F$ at the $j$ th crossing of the original curves as $\tilde{F}^{j}$. Consider the last intersection, assumed to occur at $\vec{F}^{n}$, as shown in Figure 1 for the case of two intersections. Since $\mu_{i} \Phi_{i}(1)=\mu_{i}$, the curve for the distribution with the higher mean crosses the curve for the distribution with the lower mean from below at $\tilde{F}^{n}$. Suppose, then, that $\mu_{1} \geq \mu_{2}$ and that $\mu_{1} \Phi_{1}$ intersects $\mu_{2} \Phi_{2}$ from below at $\tilde{F}^{n}$, as in Figure 1. This requires that $\mu_{1} \Phi_{1}^{\prime}\left(\tilde{F}^{n}\right)>\mu_{2} \Phi_{2}^{\prime}\left(\tilde{F}^{n}\right)$, and therefore $y_{1}\left(\tilde{F}^{n}\right)>y_{2}\left(\tilde{F}^{n}\right)$. Denoting the $(n-1)$ th intersection by $\tilde{F}^{n-1}$, the mean value theorem requires that there exists some $\bar{F}^{n-1, n} \in\left[\tilde{F}^{n-1}, \tilde{F}^{n}\right]$, such that $\mu_{1} \Phi_{1}^{\prime}\left(\bar{F}^{n-1, n}\right)=\mu_{2} \Phi_{2}^{\prime}\left(\bar{F}^{n-1, n}\right)$. By Theorem 1, equal slopes of the generalized Lorenz curves imply that

$$
\mu_{3} \Phi_{3}\left(\bar{F}^{n-1, n}\right)=p_{1} \mu_{1} \Phi_{1}\left(\bar{F}^{n-1, n}\right)+\left(1-p_{1}\right) \mu_{2} \Phi_{2}\left(\bar{F}^{n-1, n}\right)
$$

Since $\mu_{3} \Phi_{3}$ inust lie between the two original curves at $\bar{F}^{n-1, n}$ by (13), must be less than or equal to them at $\tilde{F}^{n}$ by Theorem 1 , and must be intermediate to them at $F=1, \mu_{3} \Phi_{3}$ must intersect $\mu_{1} \Phi_{1}$ from above in the interval $\left[\bar{F}^{n-1, n}, \tilde{F}^{n}\right]$, and must intersect $\mu_{2} \Phi_{2}$ from below in the interval $\left[\tilde{F}^{n}, 1\right]$. Now suppose that $\tilde{F}^{n-1} \neq 0$, implying that the original curves cross at least twice, with $\mu_{2} \Phi_{2}>\mu_{1} \Phi_{1}$ in the interval $\left[\tilde{F}^{n-1}, \tilde{F}^{n}\right]$. Then there exists some $\tilde{F}^{n-2, n-1} \in\left[\tilde{F}^{n-2}, \tilde{F}^{n-1}\right]$ at which the slopes of the two original curves are equal, requiring that $\mu_{3} \Phi_{3}$ lie between the original curves at that point. The same logic as above requires that $\mu_{3} \Phi_{3}$ must interseci $\mu_{2} \Phi_{2}$ from above in the interval $\left[\tilde{F}^{n-2}\right.$, 


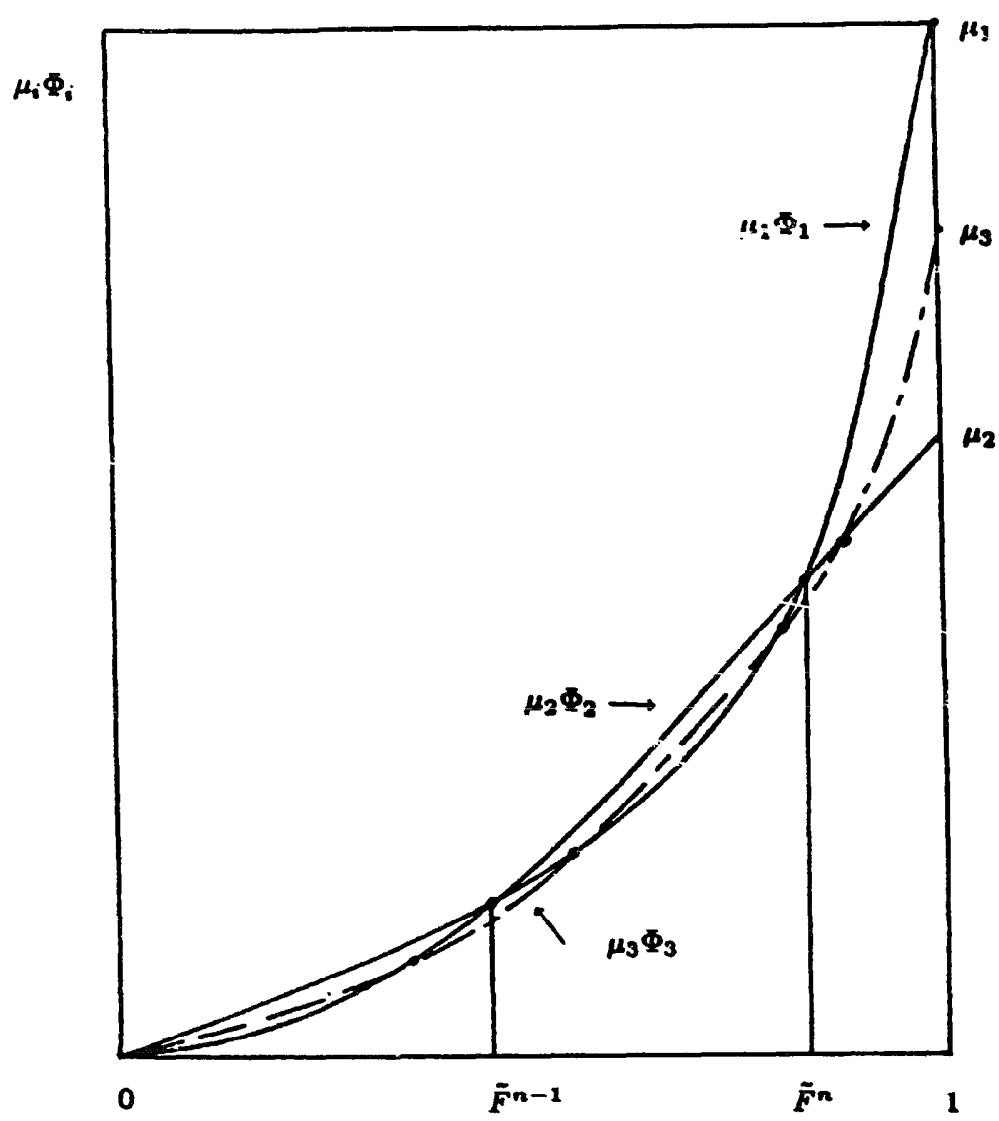

Figure 1. Generalized Lorenz curves for initial population, $\mu_{1} \Phi_{1}$, entering population, $\mu_{2} \Phi_{2}$, and combined population, $\mu_{3} \Phi_{3}$, for a hypothetical case of two intersections in original curves.

$\left.\tilde{F}^{n-1}\right]$ and must intersect $\mu_{1} \Phi_{1}$ trom below in the interval $\left[\tilde{F}^{n-1}, \tilde{F}^{n}\right]$. By induction, every additional intersection of the original curves implies that $\mu_{3} \Phi_{3}$ intersects each of the original curves at least one additional time.

It can now be shown that the curve for the combined population intersects each of the original curves exactly $n$ times, with the $n$ intersections whose existence was proven above being the only intersections. The proof follows from the following property of the generalized Lorenz curve for the combined population: If $\mu_{3} \Phi_{3}$ cuts one of the original curves from above, it cannot cut the same curve from below without first crossing the other curve. To see this, suppose there did exist such a pair of intersections, with $\mu_{3} \Phi_{3}$ crossing $\mu_{2} \Phi_{2}$ from above at some $F^{A}$, and from below at some $F^{B}$, with $\mu_{1} \Phi_{1}>$ $\mu_{2} \Phi_{2} \forall F \in\left[F^{A}, F^{B}\right]$. Then there is some $\bar{F}^{A, B} \in\left[F^{A}, F^{B}\right]$ at which 
$\mu_{3} \Phi_{3}\left(\bar{F}^{A, B}\right)<\mu_{2} \Phi_{2}\left(\bar{F}^{A, B}\right)$ and $y_{3}\left(\bar{F}^{A, B}\right)=y_{2}\left(\bar{F}^{A, B}\right)$, with $y_{3}<y_{2}$ to the left of $\bar{F}^{A, B}$ and $y_{3}>y_{2}$ to the right of $\bar{F}^{A, B}$. But given the condition in (4) that $y_{3} \in\left[y_{1}, y_{2}\right]$, these slope conditions can only hold if $y_{1} \leq y_{3}$ $<y_{2}$ to the left of $\bar{F}^{A, B}$ and $y_{1} \geq y_{3}>y_{2}$ to the right of $\bar{F}^{A, B}$. This slope reversal, in turn, can only be true if all three curves have identical slopes at $\bar{F}^{A, B}$. But from (6), $\mu_{3} \Phi_{3}$ must lie between the two original curves wherever they have equal slopes. It is thus impossible for $\mu_{3} \Phi_{3}$ to cross $\mu_{2} \Phi_{2}$ from above and then from below in any interval in which $\mu_{1} \Phi_{1}$ and $\mu_{2} \Phi_{2}$ do not cross.

By Theorem 1, $\mu_{3} \Phi_{3}$ can never intersect the higher of the two original curves in any interval. As inspection of Figure 1 makes clear, any intersection of $\mu_{3} \Phi_{3}$ with either of the original curves in addition to the $\boldsymbol{n}$ intersections whose existence was proven above would require that $\mu_{3} \Phi_{3}$ cross the lower of the two original curves from above and then below without crossing the other curve at some intermediate value. Since this has been proven impossible, the curve for the combined population must cross each of the original curves exactly $\boldsymbol{n}$ times.

The ability to identify the number of intersections of generalized Lorenz curves, and to identify the order of the curves in particular regions, can be extremely useful in analyzing the rarkings which would be given to each distribution by particular social welfare functions or inequality measures. Davies and Hoy (1985), for example, use the criterion of third-degree stochastic dominance to provide additional orderings of income distributions beyond those provided by Lorenz dominance. Their results suggest that the ability to prove that one generalized Lorenz curve lies initially below a second curve and intersects it only once would be useful information in attempting to make welfare comparisons across distributions. Unfortunately, it will be seen below that the tidy results established here for generalized Lorenz curves do not extend to standard Lorenz curves.

\section{IMPLICATIONS FOR INTERTEMPORAL COMPARISONS OF SOCIAL WELFARE}

Consider a researcher examining changes in the distribution of income over time by comparing generalized Lorenz curves for two periods. Under what conditions will the distribution indicate an unambiguous improvement in social welfare? Suppose that between the two periods the difference between departures from a population (due to deaths, retirements, or out-migration) and entrants into a population (due to births, entry into labor force, or in-migration) produce 
a "net entrants" population with a nonnegative density at every income level. ${ }^{9}$ In a growing population it may be reasonble to assume that the population in the second period can be thought of as the original population plus a group of new entrants. The results above, then, imply the following:

The second period distribution has unambiguously higher social welfare than the first period distribution if and only if the new entrants have unambiguuusly higher social welfare then the first period population. It is possible for the new entrants to have a mean income lower than the first period mean income and still have unambiguously higher social welfare, as can be seen by analogy to the uncertainty literature. It is a necessary condition for increased social welfare, however, that the minimum income among the new entrants not be less than the minimum income in the original population.

It is also possible for the second period distribution to imply unambiguously i igher social welfare in cases in which the new entrants have an unambiguously less equal distribution in the sense that the Lorenz curve for the new entrants lies everywhere below the original Lorenz curve. In this case it is a necessary condition that the new entrants' mean income exceed the original mean. If the new entrants have both a lower mean and an unambiguously less equal distribution, then the second period populations must have unambiguously lower social welfare.

\section{PROPERTIES OF LORENZ CURVES FOR COMBINED DISTRIBUTIONS}

If the two original distributions have identical means, the results of the previous sections apply directly to standard Lorenz curves. Given equal means, "expected social welfare" and "equality" may be considered formally equivalent for many purposes, with the precise sense in which this is true slarified by Atkinson (1970), Rothschild and Stiglitz (1973), and others. When mean incomes differ between two populations, then the equivalence of equality, as traditionally defined, and social welfare breaks down. One reading of Shorrock's theoretical results is that the Lorenz curve and inequality measures based on it should be abandoned in such a case in favor of Shorrocks' mean infiated generalized Lorenz curves, since the latter map directly into rankings of social welfare. As important as Shorrocks' results are, however, it

\footnotetext{
'If there are more departures than entrants at some income levels, then the density for the "entering population" will be negative at some points, and the proofs above will not hold in general.
} 
is unlikely that the concept of scale-neutral inequality will be discarded by economists or other social scientists. The independence of inequality measures from mean income has long been axiomatic in the analysis of inequality, ${ }^{10}$ and researchers will continue to analyze trends in inequality independent of changes in mean income.

What can be said, then, about the changes in inequality that will be observed when two populations are cor. ined, using the Lorenz curve as the basis for inequality comparisons ' $r$ the two populations have equal means, then all of the results de..ved above can be applied directly. For example, if $\mu_{1}=\mu_{2}$, then Theorem 2 estabishes that the Lorenz curve for the combined population must lie everywhere between the two original Losinz chines if the original Lorenz curves do not intersect. This result need nut hold for standard Lorenz curves when the means of the onig nopulations differ, however. In order to analyze the relative positions of the Lorenz curves, it is possible first to establish some results for generalized Lorenz curves which do e.tend to standard Lorenz curves. Rewriting the result in Theorem 1,

$$
\Phi_{3}(F) \leq \pi_{1} \Phi_{1}(F)+\left(1-\pi_{1}\right) \Phi_{2}(F) \forall F,
$$

where $\pi_{1}=\left(p_{1} \mu_{1}\right) / \mu_{3}$, the share of total income accounted for by the first subpopulation. Whereas the generaiized Lorenz curve for the combined population was shown to be less than or equal to a populationshare weighted average of the original generalized Lorenz curves, the standard Lorenz curve for the combined population is less than or equal to an income-share weighted average of the original Lorenz curves. This has important implications, since it means that the relative position of the Lorenz curve for the combined population will be affected in complex ways by the relative incomes of the two subpopulations.

Beyond the requirement implied by (14) that the Lorenz curve for the combined population can never lie above the maximum of the original Lorenz curves, the restrictions on the relative position of Lorenz curves are more ambiguous than the restrictions on the relative position of the generalized Lorenz curves. It is not true, for example, that the Lorenz curve for the combined population must lie between the original Lorenz curves if the original Lorenz curves do not intersect. It is also not true that the Lorenz curve for the combined population can never lie everywhere below both of the original Lorenz curves.

Is it possible to add individuals to a population so that the new combined distribution is unambiguously more equal than the original

\footnotetext{
${ }^{10}$ See Kakwani's (1980) survey, for example.
} 
distribution? Formally, under what conditions will the combined distribution, $f_{3}$, Lorenz-dominate the initial distribution, $f_{1}$, recognizing from (14) that the comined distributicis san never Lorenz-dominate both $f_{1}$ and $f_{2}$ ? The answer is that the conditions are surprisingly restrictive.

Theorem 4. If $y_{1}^{\min }>0$, then

$\Phi_{1}(F) \leq \Phi_{3}(F) \leq \Phi_{2}(F) \forall F \quad$ iff $\quad \mu_{2}=\mu_{1}$ and $\Phi_{1}(F) \leq \Phi_{2}(F) \forall F$.

The combined population's income distribution will Lorenz dominate the original population's income distribution if and only if the entering population has the same mean as the original population and the income distribution of the entering population Lorenz dominates the original population's income distribution.

Proof: The first step is to establish the necessary condition that $\mu_{2}=$ $\mu_{1}$. Since $\Phi_{i}(0)=0$ and $\Phi_{i}(1)=1$, in order for $\Phi_{3}$ to Lorenz-dominate $\Phi_{1}$, it must be true that $\Phi_{3}^{\prime} \geq \Phi_{1}^{\prime}$ as $F \rightarrow 0$ and that $\Phi_{3}^{\prime} \leq \Phi_{1}^{\prime}$ as $F$ $\rightarrow 1$. By (2) this implies that

$$
\frac{y_{3}^{\min }}{\mu_{3}} \geq \frac{y_{1}^{\min }}{\mu_{1}} \text { and } \frac{y_{3}^{\max }}{\mu_{3}} \leq \frac{y_{1}^{\max }}{\mu_{1}} .
$$

The addition of $f_{2}$ implies the introduction of new individuals to a population while leaving the incomes of the original members unchanged, so it must be the case that

$$
y_{3}^{\min } \leq y_{1}^{\min } \text { and } y_{3}^{\max } \geq y_{1}^{\max } .
$$

From the inequality conditions in (16) it is clear that the two conditions in (15) cannot both be satisfied for any $\mu_{3} \neq \mu_{1}$ as long as $y_{1}^{\min }>$ 0."

Several implications of (15) and (16) are worth noting. If $\mu_{3}>\mu_{1}$ (implying $\mu_{2}>\mu_{1}$ ), (16) requires that $y_{3}^{\min } / \mu_{3}<y_{1}^{\min } / \mu_{1}$, implying that $\Phi_{3}$ must lie below $\Phi_{1}$ out of the origin. By the same logic, if $\mu_{3}<$ $\mu_{1}$, the new Lorenz curve must lie below the original Lorenz curves as $F \rightarrow 1$. If the extreme incomes remain constant, a change in the

\footnotetext{
"If $y_{i}^{\min }=0$, it is possible to raise the mean without lowering the share of income to the poorest individuals in the population, since their shares are already at zero. This introduces additional conditions for !orenz dominance, involving Lorenz. curves which initially coincide with the horizontal axis. The result is similar to the perverse properties Lorenz curves exhibit when negative incomes are included. We therefore extend the usual restriction against negative incomes to exclude incomes at exactly zero as well.
} 
mean must imply that $\Phi_{3}$ intersects $\Phi_{1}$. Since the slopes at the corners are the extreme incomes divided by the meas , the slopes at both $F \rightarrow$ 0 and $F \rightarrow 1$ must change in the same direction when the mean changes if $y^{\text {min }}$ and $y^{\text {max }}$ remain constant. But since $\Phi_{3}=\Phi_{1}$ at $F=0$ and $F$ $=1$, the slope of $\Phi_{3}$ cannot be greater than (less than) the slope of $\Phi_{1}$ at both end points unless $\Phi_{3}$ intersects $\Phi_{1}$. Specifically, if the extreme incomes remain conistant and $\mu_{3}>\mu_{1}$, the first intersection must be $\Phi_{1}$ intersecting $\Phi_{3}$ from below. If the extreme incomes remain constant and $\mu_{3}<\mu_{1}$, the last intersection must be $\Phi_{1}$ intersecting $\Phi_{3}$ from above. If either $y_{3}^{\min }$ or $y_{3}^{\max }$ lies outside the range $\left[y_{1}^{\min }, y_{1}^{\max }\right]$, it is possible for the new Lorenz curve to lie everywhere below the original Lorenz curve. What must be true if $\mu_{3} \neq \mu_{1}$ is that $\Phi_{3}(F)<$ $\Phi_{1}(F)$ over some range of $F$. This analysis tells us not only the conditions under which the combined distribution will Lorenz dominate the original distribution, but also about the relative positions of the Lorenz curves in the case of intersections. As pointed out above in the case of generalized Lorenz curves, knowledge that one Lorenz curve must lie below a second Lorenz curve out of the origin can be useful in assessing the rankings which would be given by different inequality measures.

Given equal means, it follows directly from (10) that

$$
\Phi_{1}(F) \leq \Phi_{3}(F) \leq \Phi_{2}(F) \forall F \quad \text { iff } \quad \Phi_{1}(F) \leq \Phi_{2}(F) \forall F .
$$

Given equal means, it is hoth necessary and sufficient that $f_{2}$ Lorenz doninate $f_{1}$ in order that $f_{3}$ Lorenz dominate $f_{1}$. Since we have proven the necessary condition that the two populations have the same mean, the proof of Theorem 4 is complete.

Theorem 4 demonstrates that the restrictions on the relative position of the generalized Lorenz curves established in Theorems 2 and 3 do not extend directly to standard Lorenz curves. In contrast to the result for generalized Lorenz curves, there is no necessary relationship between the number of intersections of $\Phi_{1}$ and $\Phi_{2}$ and the number of intersections of $\Phi_{1}$ and $\Phi_{3}$, except when $\mu_{1}=\mu_{2}$. Both parts of the proof of Theorem 3 on intersections break down for standard Lorenz curves. First, it is not the case for Lorenz curves that equal slopes of the original curves require that the combined curve be intermediate to the original curves at that point. We therefore cannot establish that an intersection in the original curves implies that the curve for the combined distribution intersects both original curves. Second, there is no restriction that the slope of the Lorenz curve for the combined distribution must be intermediate to the slopes of the original Lorenz curves. The restrictions which hold are that $y_{3}(F) \in\left[y_{1}(F), y_{2}(F)\right]$ and $\mu_{3} \epsilon$ 
$\left[\mu_{1}, \mu_{2}\right]$. Since the slope of the Lorenz curve is $y_{i} / \mu_{i}$, it is possible for the slope of $\Phi_{3}$ to be either greater or smaller than the slopes of both $\Phi_{1}$ and $\Phi_{2}$. This means the restriction derived in the proof of Theorem 3 that the combined curve cannot cross another curve from above and below consecutively does not hold in the case of Lorenz curves, except when $\mu_{1}=\mu_{2}$.

Unlike the case for generalized Lorenz curves, it is clearly possible for the Lorenz curve for the combined distribution to lie everywhere below both of the riginal Lorenz curves. In other words, while the combined population can never have unambiguously lower expected social welfare than both of the original populations, the combined population can have an unambiguously less equal distribution than both of the original populations. The simplest example is the case in whicn the two populations are each purely egalitarian but have different means. The conivined distribution must be unambigously more unequal than either of the originai distributions, with the Lorenz curve (consisting simply of two line segments with slopes equal to the ratio of the subpopulation mean to the combined mean) lying everywhere below the original egalitarian Lorenz curves. The combined population will have unambiguously higher (lower) expected social welfare than the distribution with the lower (higher) mean, however, a simple demonstration of the nonequivalence of inequality rankings and social welfare rankings under different means.

\section{IMPLICATIONS FOR INEQUALITY COMPARISONS}

As above, it is instructive to consider the implications of these results for analysis of changes in inequality in a population over time, continuing to assume that the second period population can be thought of as the original population plus a group of new entrants. By Theorem 4 , if the population of new entrants has a mean income different than the initial mean income, then either the Lorenz curve for the second period lies everyw here below the origing! Lorenz curve or the Lorenz curve for the second period intersects the original Lorenz curve. Even if progressive income transiors occur between periods, the effect of the new entrants creates a tendency to reject the hypothesis of a decline in inequality. ${ }^{12}$

The results also apply to changes which are not simply the result of

\footnotetext{
${ }^{12}$ See Lam (1986) for a more comple $e$ analysis of the effects on inequality comparisons of income differentials in fertiity. Morley (1981) also points out the confusing effects of new entrants into the population an standard nequality measures.
} 
new persons entering the population. Consider the transfer of an individual from one income level to another, due, for example, to ruralurban migration. ${ }^{13}$ It is easy to prove with generalized Lorenz curves that a transfer of an individual upward (downward) in the income distribution will unambiguously increase (decrease) social welfare for any concave social welfare function, with the ex post generalized Lorenz curve dominating (dominated by) the original generalized Lorenz curve. By Theorem 4, however, the transfer of an individual in either direction will always produce intersecting Lorenz curves if the extreme incomes in the distribution remain constant. This implies that some inequality measure will always find the upward mobility of an individual disequalizing, in spite of the fact that it is an unambiguous Pareto improvement. Swamy (1967), Robinson (1976), Fields (1979), and Stark and Yitzhaki (1982) provide examples of this effect with particular inequality measures, and show that sufficiently large transfers upward will eventual' have an equalizing effect. The more general results here demonstrate $t$ such transfers will alwavs produce intersecting Lorenz curves, so that even though a particular measure shows a decline in inequality, there always exists some alternative measure which will show an increase in inequality.

\section{COMPARING INCOME DISTRIBUTIONS OF FATHERS AND SONS IN BRAZIL}

The theoretical results proven above can be illustrated by considering what would happen to the distribition of income across generations if every son received the same income as his father. The specific case considered is Brazil, a developing country which has experienced relatively high rates of population growth, has been the focus of a major debate over treiids in inequality, and has excellent microdata. Without making any pretext of constructing a complete model of intergenerational transmission of inequality, it is instructive simply to assign sons

\footnotetext{
${ }^{13} \mathrm{~A}$ number of economists, including Swamy (1967), Robinson (1976), Fields (1979), and Stark and Yitzhaki (1982) have demonstrated that transferring individuals from a low income class to a higher income class can cause inequality measures to show initial increases in inequality, followed by decreases in inequality as the higher income class increases in relative size. Fields (1979) shows that in a two-sector economy a transfer of an individual from the poor sector to the rich sector will produce a Lorenz curve which intersects the original Lorenz curve. His example is easily understood given the results above. It is a simple special case of the result established in Theorern 4 that if the minimum and maximum incomes remain constant between period 1 and period 2, any change in the mean income between the two periods implies that the Lorenz curves for the two periods intersect.
} 
the income of their fathers and cornpare the Lorenz curves and generalized Lorenz curves for the fathers' and sons' income distributions.

Assigning fathers' incomes to their sons would not generate a significant change in the distribution of income in the absence of a systematic relationship between income and fertility. The exercise becomes interesting because of the fairly substantial differences in fertility across income levels in Brazil. As shown in Lam (1986), for example, Brazilian men in the highest income quintile of the 40-45 age group averaged 4.9 total births, while men in the lowest income quintile of the same age group avera ged 6.2 total births. The experiment here, then, is simply to compare the income distribution for men to the same distribution when it is weighted by the number of sons born to each man. ${ }^{14}$

Figure 2 plots the generalized Lorenz curves for fathers and sons in Brazil based on the 1976 income ciistribution for men aged 30-45 and for boys 0-15 when they are assigned the incomes of their fathers. ${ }^{15}$ As can be seen directly from the $u_{1}$ pper corner of the generalized Lorenz curves, the fathers' distribution has a higher mean than the sons, the natural result of the negative relationship between father's income and fertility shown in Lam (1986). The negative correlation between income and fertility is sufficiently strong, in fact, to cause the generalized Lorenz curve for fathers to lie everywhere above the generaiized Lorenz curve for sons. Following Shorrocks, this implies that the fathers' distribution will have higher expected social welfare for any $S$-corcave social welfare function. Whether it is appropriate to infer an unambiguous deterioration in social 'welfare purely as a result of fertility behavior is obviously problematic. What Figure 2 demonstrates is the effects on generalized Lorenz curves of differential fertility alone, with neither progressive nor regressive iransfers across the population of sons.

Figure 3 shows the conventional Lorenz curves for Brazilian fathe: and sons, based on the same distributions used in Figure 2. Unlike t.e generalized Lorenz curves, the conventional Lorenz curves intersec:, implying that no unambiguous inequality rankings can be made between the two distributions. The theoretical results derived above make

\footnotetext{
${ }^{14}$ The number of sons is used, rather thin the number of children, in order to approximate a second generation male population. A simil ir exercise could be applied to couples and all children, but subtleties in the treatment of female labor force particiption greatly complicate such an exercise.

${ }^{15}$ The data are taken from the $1976 \mathrm{~F}$, squisa Nacional de Amostra Domiciliar (PNAD), a survey of 99,167 households conducted by thc Instituto Brasileiro de Geogratia e Estatistica.
} 


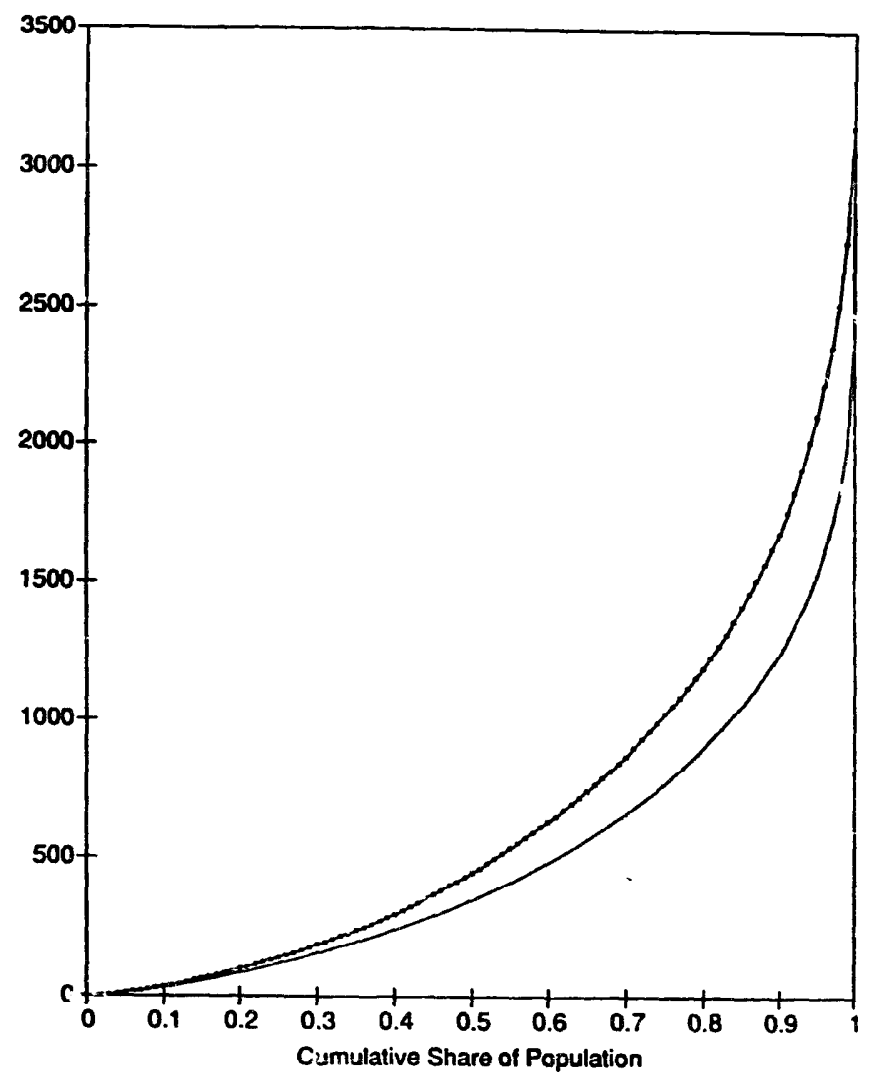

Figure 2. Generalized Lorenz curves for Brazilian men aged 30-45 (-) and for Brazilian boys aged 0-15 (-) assigned income of father. (Source: 1976 PNAD.)

this outcome entirely transparent. Although every man in the original distribution was not necessarily a father, implying that some points in the original density may have gone unreplaced in the second generation, in fact the experiment provides a very close approximation to the hypothetical mixing of distributions analyzed formally above. The extreme incomes for sons are the same as the extreme incomes for fatheis. Since the mean falls across generations. the results above require not only that the Lorenz curves must cross, but require also that the sons' Lorenz curve lies above the fathers' curve at the bottom and lies below the fathers' curve at the top of the distribution. In Figure 3 , these requirements are satisfied in the simplest possible way, by a single intersection. This single intersection is not a theoretical restriction, and any number of intersections would have been consistent with the basic facts of the distribution, as long as the sons' Lorenz curve started above and ended below the fathers' Lorenz curve. The fact that 


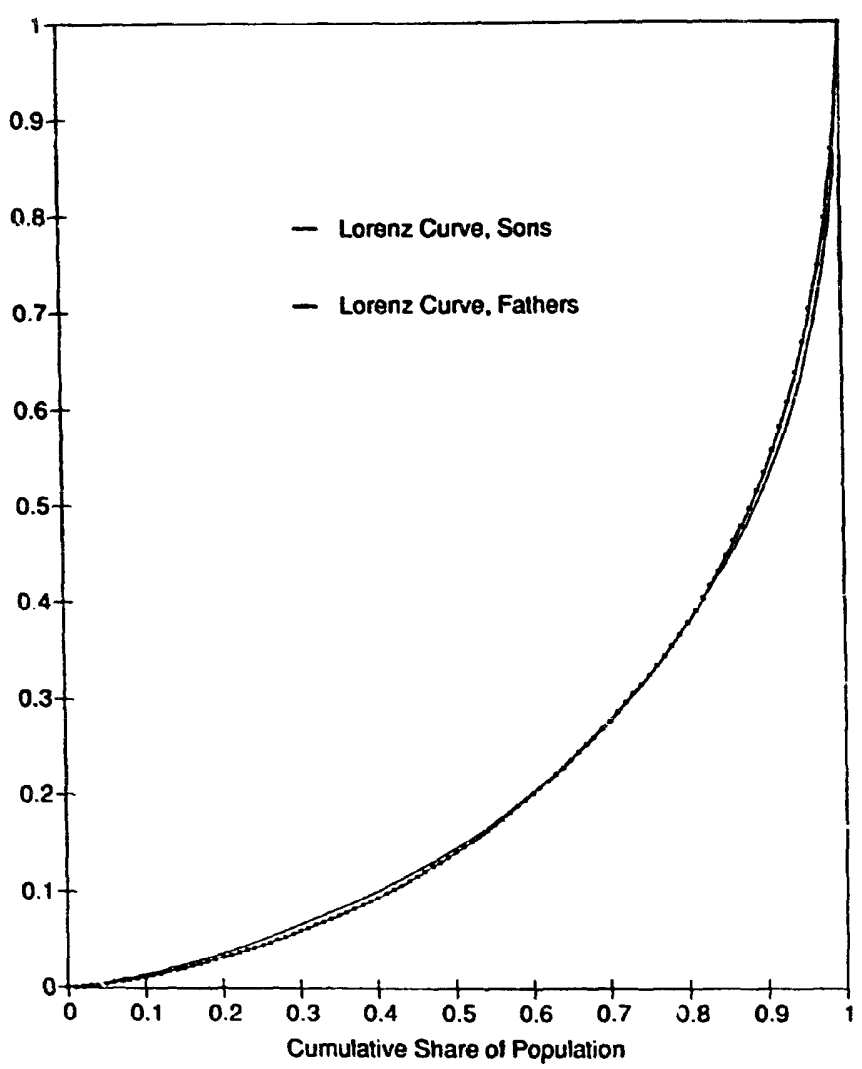

Figure 3. Lorenz curves for Brazilian men aged 30-45 (-) and for Brazilian boys aged 0-15 (-) assigned income of father. (Source: 1976 PNAD.)

the sons' Lorenz curve initially cuts the fathers' Lorenz curve from below means that the sons' distribution is more likely to be judged more equal by inequality measures that are highly sensitive to transfers at the bottom of the distribution, such as the log variance or Atkinson's index with a high degree of "relative inequality aversion."

It goes without saying that the intergenerational transmission of inequality is far more complex than sons taking on the incomes of their fathers. Figures 2 and 3 provide a warning, however, of the changes in income distributions that can be induced purely by demographic factors. In the $t$, pical case in which lower income is associated with higher fertility, Figure 2 suggests that real wage increases, which would lead to generalized Lorenz dominance in an unchanging population, may be overcome by the tendency for differential fertility to lower the generaiized Lorenz curve. Figure 3 similarly suggests that progressive or regressive transfers, which would imply 
Lorenz dominance of one period over another in an unchanging population, may be lost in the intersecting Lorenz curves that are created by differential fertility.

\section{CONCLUSIONS}

Lorenz curves have received renewed attention as a basis for inequality comparisons by proofs of the equivalence between rankings of income distributions by Lorenz dominance and rankings by concave social welfare functions. This paper demonstrates that caution should be used in such comparisons due to the sensitivity of Lorenz curves to changes in population composition. The paper establishes several general properties of Lorenz. curves and generalized Lorenz curves produced from combinations of distributions. For generalized Lorenz curves, the conditions on the curve for the combined distribution are quite orderly. The generalized Lorenz curve for the combined distribution is always less than or equal to a weighted average of the original curves, always lies between the original curves when the original curves do not intersect, and intersects each of the original curves exactly the same number of times as the original curves intersect.

The results for standard Lorenz curves are less regular, and reveal the inherently ambiguous nature of intertemporal inequality comparisons. The paper proves that the Lorenz curve for the combined distribution is always less than or equal to the highest of the two original Lorenz curves. Beyond this, few restrictions on the Lorenz curves can be made when the means of the original populations differ. An important result is that the combined distribution will Lorenz dominate the initial distribution if and only if the entering population has an identical mean income and itself Lorenz dominates the initial distribution. If the highest and lowest incomes in the initial population remain constant, any change in the population which alters mean income will produce a new Lorenz curve which intersects the original Lorenz curve.

Income distributions for fathers and sons in Brazil demonstrate the theoretical results. Assigning sons the incomes of their fathers, the second generation generalized Lorenz curve lies everywinere below the generalized Lorenz curve for fathers. The standard Lorenz curves for the two generations intersect, with the sons' curve lying initially above the fathers' curve. The Lorenz curves behave exactly as the theoretical results require, providing a simple illustration of the purely demographic component of intertemporal changes in inequality.

The results imply that intertemporal inequality comparisons in the 
presence of changing population composition must be viewed with caution. Although the population may experience progressive or regressive transfers which have a straightforward welfare interpretation and which themselves produce unambiguous effects on Lorenz curves, contemporaneous changes in population composition may have confounding effects on the Lorenz curves which obscure the transfer effects and make welfare comparisons from standard inequality measures impossible.

\section{REFERENCES}

Atkinson, Anthony B. (1970) On the Measurement of Inequality, Journal of Economic Theory 2:244-263.

Dasgupta, P., Sen, A.K., and Starrett, D. (1973) Notes on the Measurement of Inequality, journal of Economic Theory 6:180-187.

Davies, James, and Hoy, Michael (1985) Comparing Income Distributions Under Aversion to Downside Inequality, Centre for the Study of International Economic Relations Working Pafer No. 8521C, Department of Economics, University of Westem Ontario, London, Canada.

Fields, Gary S. (1979) A Welfare Economic Approach to Growth and Distribution in the Dual Economy, Quarterly Journal of Economics 93(3):327-353.

Fields, Gary S., and Fei, John C.H. (1978) On Inequality Comparisons, Econometrica 46(2):303316.

Kakwani, Nanak C. (1980) Income Inequality and Poverty: Methods of Estimation and Policy Applications. Oxford, U.K.: Oxford University Press.

Lam, David (1986) The Dynamics of Population Growth, Differential Fertility, and Inequality, American Economic Review 76(5):1103-1116.

(1987) Distribution Issues in the Relationship Between Population Growth and Economic Development. In Population Growth and Economic Development: Issues and Evidence (D. Gale Johnson and Ronald D. Lee, Eds.). Madison, WI: University of Wisconsin Press.

Morley, Samuel (1981) The Effect of Changes in the Population on Several Measures of Income Distribution, American Economic Review 71(3):285-294.

Nerlove, Marc, Razin, Assaf, and Sadka, Efraim (1987) Household and Economy: Welfare Economics of Endogenous Fertility. Boston: Academic.

Newbery, D. (1970) A Theorem on the Measurement of Inequality, Journal of Economic Theory 2:264-265.

Robinson, Sherman (1976) A Note on the U Hypothesis Relating Income Inequality and Economic Development, American Economic Review 66(3):437-440.

Rothschild, Michael, and Stiglitz, Joseph E. (1973) Some Futher Results on the Measurement of Inequality, Journal of Economic Theory 8:188-204.

Satchell, S.E. (1984) Source and Subgroup Decomposition Inequalities for the Lorenz Curve, Department of Economics Discussion Paper No. 252, University of Essex.

Sheshinski, E. (1972) Relation Between a Social Welfare Function and the Gini Index of Income Inequality, Journal of Economic Theory 4:98-100.

Shorrocks, Anthony F. (1983) Ranking Income Distributions, Economica 50:3-17.

Stark, Oded, and Yitzhaki, Shlomo (1982) Migration, Growth, Distribution, and Welfare, Economics Letters 10:243-249.

Swamy, Subramanian (1967) Structural Changes and the Distribution of Income by Size: The Case of India, Review of Income and Wealth 13:155-174. 